\title{
FORO REALIZADO EN EL HOSPITAL DE SAN JOSÉ SOBRE LA LEY 1122 DE 2007 Reforma de la seguridad social en salud
}

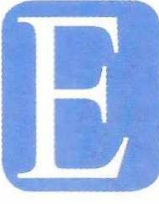

113 de marzo de 2007 se realizó en el auditorio Guillermo Fergusson del Hospital de San José el foro sobre la reforma de la seguridad social (ley 1122 de 2007) con la participación del Señor Ministro de la Protección Social Dr. Diego Palacio y los doctores Jorge Gómez Cusnir presidente de la Sociedad de Cirugía de Bogotá, Hospital de San José, Juan Carlos Giraldo presidente de la Asociación Colombiana de Hospitales y Clínicas, Juan Manuel Diaz-Granados presidente de ACEMI y Juan Carlos Trujillo de Hart director general de análisis y política de recursos humanos del Ministerio de la Protección Social.

El foro se dividió en tres partes, en la primera el Dr. Jorge Gómez Cusnir, presentó los resultados del análisis y la posición de la Sociedad de Cirugía frente a la ley $1122 \mathrm{y}$ el proyecto de ley sobre talento humano, en la segunda el Dr. Diego Palacio, hace un análisis de las ventajas que tienen los citados ley y proyecto, para los diferentes actores inmersos en el sistema de Seguridad Social en Salud y en la tercera se resolvieron las preguntas de los asistentes.

E1 Dr. Jorge Gómez manifestó que la ley 1122 pretende mejorar los alcances de la ley 100 de 1993, hace un abordaje integral de los grandes problemas de salud identificando los siguientes:

- Cobertura total.

- Falta de regulación del mercado.

- Gestión de la calidad.

- Flujo de recursos.

- Debilidad en el control.

- Debilidad en salud pública.

- Déficit en la estructura de los sistemas de información.
Con base en lo anterior resalta como aspectos positivos:

- El usuario sigue siendo el eje central del sistema.

- Se preservan los principios rectores de la ley 100 de 1993.

- Gran importancia a la cobertura.

- Se aclara el panorama de gestión sanitaria territorial y de las ARS en aseguramiento.

- La creación de la comisión de regulación en salud CRES.

- El mantenimiento de la estructura básica del sistema.

- Fortalecimiento de la inspección, vigilancia y control.

- Asigna nuevas funciones a la Superintendencia Nacional de Salud.

- Pago anticipado a las IPS para garantizar el flujo de recursos.

Como aspectos negativos enuncia que:

- Prevalece la inequidad sanitaria en el aseguramiento.

- Persisten las diferencias entre el régimen contributivo y el subsidiado.

- Hay un incremento en servicios no POS.

- Para las IPS privadas hay un riesgo con el incremento de la contratación con las públicas al $60 \%$ en el régimen subsidiado.

Un aspecto que merece una especial revisión es lo relacionado con la integración vertical del 30\%.

Con respecto al proyecto de ley de talento humano en salud el Dr. Gómez hace referencia a la creación del organismo que establece todas las disposiciones relacionadas con el tema y los entes encargados de apoyarlo. Resalta: 
- Los comités de talento humano en salud.

- El papel fundamental de los colegios en los procesos de recertificación profesional.

- El énfasis que hace el proyecto en los centros de práctica y el desarrollo de los convenios docencia-servicio.

- Los beneficios del hospital universitario y el análisis de los programas de formación en salud en términos de cantidad y calidad.

-En cuanto al ejercicio de las profesiones en el área de la salud, se regulan los siguientes aspectos:

- Requisitos para el ejercicio.

- Registro único nacional.

- Recertificación en la cual se asigna un papel importante a los colegios de profesionales y se genera el interrogante acerca de la participación de la educación superior en este proceso.

- Servicio social obligatorio.

-En lo referente a las políticas para el desempeño el proyecto de ley contempla:

- Manual de tarifas mínimas.

- Programas de estímulos e incentivos.

- Becas crédito.

- Prestación del servicio basados en los principios y valores.

Con base en los anteriores planteamientos el Dr. Diego Palacio analiza los principales aspectos de la ley. En primera instancia expresa la convicción que tiene el Ministerio de la Protección Social de que el 2007 es un excelente año para el sector no solo por la ley 1122 y el proyecto de ley de talento humano en salud, sino también por el decreto de regulación de las EPS que les permite organizarse como un verdadero asegurador con las reservas respectivas y entrar en un proceso de habilitación que ha demostrado sus bondades cuando ha sido realizado por las ARS y las IPS, adicionalmente el tema de acreditación voluntaria ha tenido gran aceptación en el sector. Prueba de ello es que cerca de 200 IPS han iniciado el proceso y como incentivo para ellas el Ministerio está buscando que las entidades acreditadas tengan el tratamiento de zona franca, lo que les permite

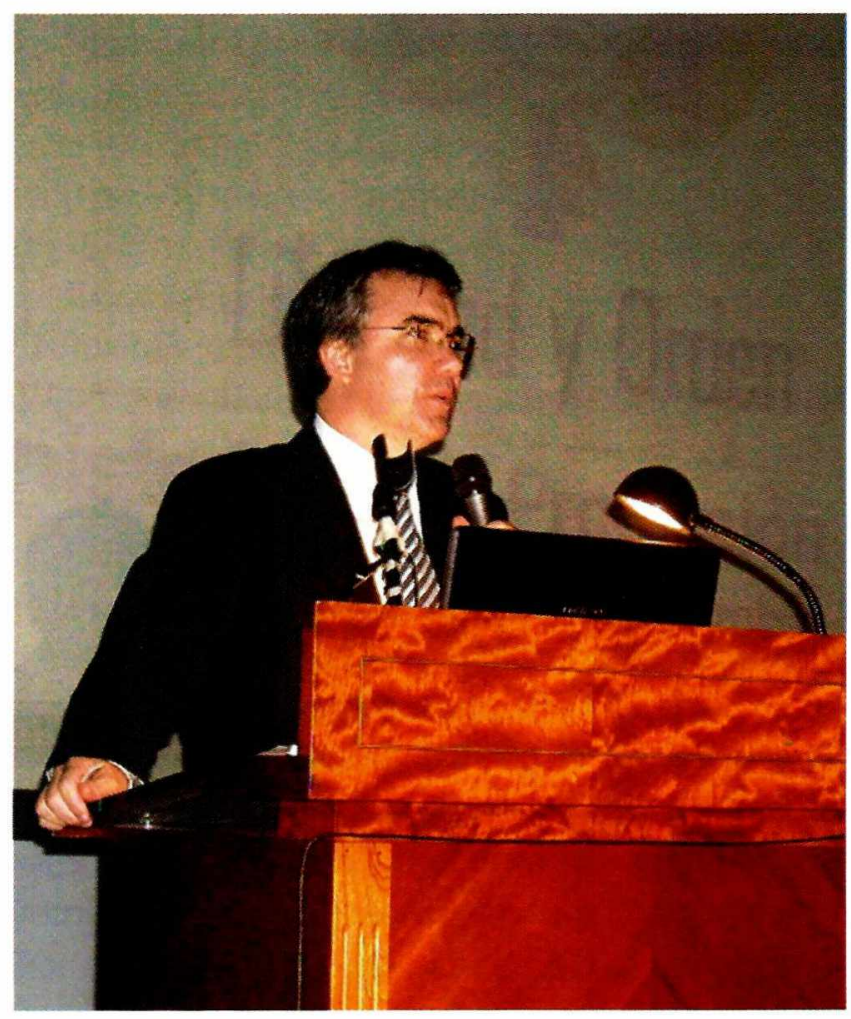

Dr. Diego Palacio Betancourt, Ministro de la Protección Social durante la intervención en el Foro sobre la Reforma de Seguridad Social en Salud, realizado en el Hospital de San José el 13 de marzo de 2007.

agilizar las operaciones para exportar salud. Otros aspectos positivos a contemplar son:

- La solución a la problemática del seguro social.

- El diseño e implementación de estrategias para manejar el alto costo.

- Se está trabajando para que las entidades acreditadas tengan préstamos con tres años muertos y bajas tasas de interés.

- La sanción de la ley de recursos humanos en Salud.

-Durante este año se espera realizar la reglamentación de la ley 1122 cuyos conceptos centrales son:

\section{Dirección y regulación con base en la Comisión de} Regulación en Salud (CRES), cuyas funciones son:

- Regulación de parámetros técnicos del sistema.

- Establecer el monto de la UPC.

- Regular las cuotas moderadoras y copagos. 
- Establecer el contenido de los POS.

- Listado de medicamentos.

2. Financiación: se aumentará la cotización en salud del 12 al 12.5\%. Este $0.5 \%$ estará a cargo de los empleadores y se espera que se aumenten los recursos, haya mayor velocidad en el flujo de los mismos y contención del costo para que en el año 2009 la cobertura sea universal.

\section{Aseguramiento:}

-Se asigna subsidio pleno para las personas clasificadas en SISBEN I y II y uno parcial para las personas clasificadas en el III asegurando la cobertura de riesgos que no puede ser realizada por la familia. En este punto es necesario hacer el análisis conceptual de la equidad para dejar en claro que no es sinónimo de igualdad.

-La restricción de la integración vertical de IPS que tiene como fin asegurar que no se presente una posición dominante, lo cual es lesivo en cualquier sistema, por lo tanto se limita la contratación con su red propia a un $30 \%$.

-La reforma elimina barreras de acceso a los servicios de salud y se disminuyen los períodos de carencia a 26 semanas.

-Prestación de servicios: el Ministerio define los requisitos para la habilitación y establece las medidas para evitar la selección adversa. Los usuarios tendrán libertad para cambiar de EPS.

4.Salud pública: se establece el plan de desarrollo para cada cuatrienio.

5. Fortalecimiento de vigilancia y control: se crea el defensor del usuario en salud, vocero de los afiliados a las EPS.

Con respecto a la ley 024 de 2004 el señor Ministro resalta los siguientes aspectos:

-Se crea el Consejo de talento humano en salud.
-Se establece el registro único nacional de talento humano, el cual se convierte en una herramienta importante para establecer políticas al respecto.

-Certificación y recertificación cada cinco años para lo cual se contará con el apoyo de los colegios profesionales.

-Se dejan claramente establecidos los requisitos que deben tener los escenarios de práctica para brindar docencia de óptima calidad.

-Se establece un capitulo de estímulos e incentivos para el talento humano en salud.

-Se determinan principios éticos y bioéticos para la prestación de los servicios, lo cual está directamente relacionado con la calidad.

Con base en los anteriores planteamientos se procede a dar respuesta a las preguntas del público.

\section{1.jCómo se explica la limitación para los asegu- radores al $30 \%$ y se amplía al $60 \%$ para entes públicos?}

Al respecto el Sr. Ministro comenta que lo más importante en ese artículo es tener claras las reglas de juego y la pretensión es que ninguno de los actores pueda tener posición dominante, se busca que la red pública en igualdad de condiciones tenga posibilidad de acceso a recursos. El Dr. Giraldo comenta que las dos medidas apuntan a dos políticas diferentes: en el caso de los prestadores se busca garantizar un mercado y por lo tanto la supervivencia de las entidades públicas y además está condicionada la contratación a atributos como la disponibilidad y la calidad, si no cuentan con ellas se podrá contratar con entidades privadas previa autorización del Ministerio de la protección social. En el caso de los aseguradores cualquier integración vertical debe ser censurada.

E1 Dr. Díaz Granados representante de ACEMI aclara que el negocio de las EPS es de aseguramiento, lo cual es una asunción de riesgos frente a una contribución y se recibe en función de lo que se necesita. El asegura- 
dor selecciona el riesgo, pero en salud no es posible porque no se puede rechazar ninguna solicitud, una segunda característica es que el asegurador debe fijar el precio o establecer la prima, tampoco procede porque la UPC la fijará el CRES, la tercera característica que es el diseño del producto tampoco es posible en salud, el POS fue diseñado y cuantificado en 1994 y en la actualidad hay una presión para convertir el plan de beneficios en ilimitado. Adicionalmente el derecho de la salud asociado con el de la vida se convierte en tutelable, como también sucede cuando se vincula a la integridad y a la dignidad. Por lo tanto, hay que repensar si los recursos disponibles son suficientes porque finalmente lo único que le queda a las EPS como asegurador es administrarlos.

\section{2.¿Porqué no se limita la creación de facultades de medicina?}

E1 Dr. Juan Carlos Trujillo recuerda al auditorio que la ley 30 de 1992 establece el principio de autonomía universitaria, por lo tanto el Ministerio de educación no tiene las herramientas para limitar el surgimiento de nuevos programas. En salud el Ministerio como mecanismo de control está verificando escenarios de práctica como una forma de asegurar la calidad en la formación. Al respecto el Sr. Ministro plantea que no hay ni la fuerza política ni los elementos jurídicos para limitar el número de facultades, pero en efecto es un problema para los próximos diez años.

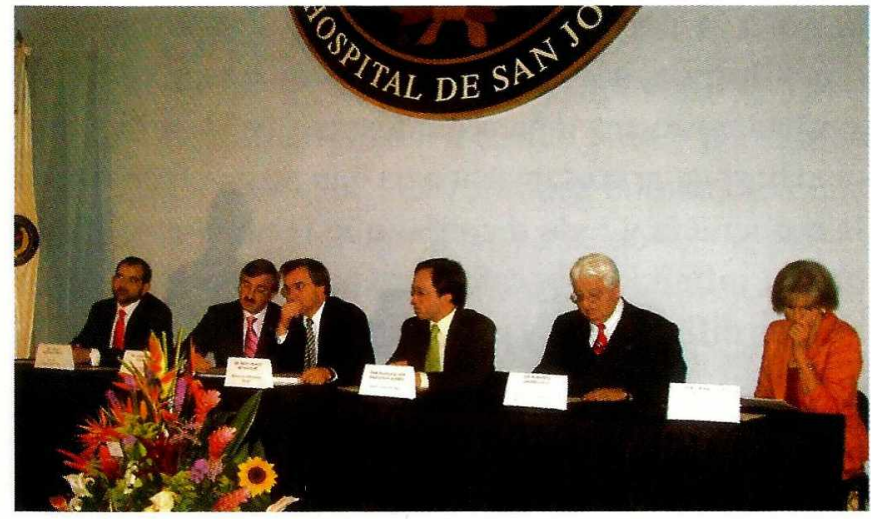

De izquierda a derecha aparecen los doctores Carlos Humberto Pérez, Director del Hospital de San José, Jorge Gómez Cusnir, Presidente de la Sociedad de Cirugía de Bogotá, Hospital de San José, Diego Palacio Betancourt, Ministro de la Protección Social, Juan Carlos Trujillo de Hart, director general de análisis y política de recursos humanos del Ministerio de la Portección Social, Roberto Jaramillos Urioechea, Rector de la Fundación Universitaria de Ciencias de la Salud y Laima Didzilius G. directora del Hospital Infantil Universitario de San José.
3. iTeniendo en cuenta los índices de mortalidad y morbilidad que tiene el país en cáncer de cervix sería posible dirigir un programa de incentivos y estímulos para las facultades de citohistología? ¿Podría incluirse esta profesión en el servicio social obligatorio?

El Dr. Trujillo comenta que una de las disciplinas que se debe fortalecer es la citohistología. Con respecto al servicio social obligatorio, está contemplado desde 1981 para todos los profesionales de la salud pero solo esta reglamentado para cuatro profesiones y se puede incluir en los estudios que se están realizando en la actualidad.

\section{4.¿Cuál es el aporte de la ley 1122 a la salud mental?}

El ministro comenta que hay dos temas, salud pública y salud mental, donde se abre la posibilidad de incorporar en los planes de salud elementos afines a la salud mental, y el Dr. Trujillo comenta que a futuro serán tenidos en cuenta para certificación y recertificaciòn al igual que en la revisión de currículos para registro calificado.

5.La ley 1122 establece un organismo técnico para los aspectos financieros ise van a tener igualmente mecanismos de control de calidad para efectos de establecer planes de mejoramiento?

El Dr. Giraldo comenta que es un logro seguir manteniendo el sistema de aseguramiento de la calidad porque finalmente los ganadores netos son los usuarios, pero lo mas importante en que el articulo $\mathrm{N}^{\circ} 2$ de evaluación de resultados contemple indicadores que midan el mejoramiento y mantenimiento en el nivel de salud de la población colombiana.

\section{6. ¿Qué va a pasar con las cooperativas en el marco de la ley de talento humano?}

E1 Dr. Palacio plantea que desde el punto de vista legal las cooperativas no pueden ser intermediarios, se está a punto de emitir un decreto que limite o termine con una práctica que no le conviene a ninguna institución. 


\section{7.¿Qué implicación económica tiene el reajuste de la UPC y la disminución en el tiempo de carencia?}

El ministro comenta que este no es un punto de fácil discusión, el congreso planteó que no había lugar a la misma y ante el planteamiento de si el piso tarifario para las aseguradoras es inexequible el Dr. Giraldo comenta que hasta el momento no se conoce el texto completo del fallo porque quedó como séptima función de la comisión de regulación.

\section{8.¿Que planes viene adelantando el Ministerio} frente a la limitación que tienen los entes territoriales con el déficit de capacidad técnica?

La condición para contratar con entidades complementarias es tener el diagnóstico de disponibilidad de la red pública.

\section{9.¿Cuál es el propósito de los colegios profesionales?}

El Dr. Trujillo aclara que la ley señala que el gobierno puede delegar algunas actividades en los colegios, pero al respecto debe tenerse en cuenta:

-La delegación de funciones no implica delegación de responsabilidades.

-La recertificación busca garantizar la calidad pero el proceso no es exclusivo de los colegios, se pretende que para la realización del mismo se integren con los formadores. Frente al costo, la ley consigna que debe ser de cinco salarios mínimos diarios vigentes, pero anexo a este proceso hay un capitulo de formación continua a la cual se asignará puntaje al igual que a la investigación.

\section{0.¿Qué incentivos se establecen en la ley 1122 para los hospitales de IV nivel de complejidad?}

El Dr. Trujillo comenta que el hospital universitario necesariamente tiene que estar acreditado y por tanto accederá a estos beneficios. Si es público existe la posibilidad de que se le autorice la emisión de estampillas para que perciba ingresos por esta vía; otro incentivo es el no cobro de la tasa de vigilancia.

\section{1. ¿Que efectos tiene el incremento patrimonial de las EPS sobre los afiliados?}

El ministro manifiesta que lo que se espera es que el incremento de los patrimonios de la EPS se haga con recursos de los accionistas y no de los usuarios. Al respecto el Dr. Díaz Granados aclara que es necesario establecer la diferencia entre el concepto de solvencia estática y solvencia dinámica porque hay que tener en cuenta el cuándo y el cuánto y a ello es necesario anexar los costos que se generan por tutelas y por período de espera.

En la intervención final el señor ministro expresa que no le queda duda sobre la bondad de la ley porque permite racionalizar mucho más los recursos existentes, pero depende en gran medida de la reglamentación y a continuación hace una reflexión sobre la situación de crisis que vivió el hospital de San José hace unos años y la compara con el crecimiento actual, para llegar a la conclusión de que en Colombia no hay crisis hospitalaria sino crisis de gerencia. Invita a los asistentes a avanzar para demostrar que la administración hospitalaria es una ciencia que no da espacio para la burocracia y pide recordar que la única necesidad básica infinita es la salud porque siempre existirán nuevas dificultades y expectativas.

* Relatora Lic. Ana Julia Carrillo A. Profesora de enfermería Fundación Universitaria de Ciencias de la Salud.
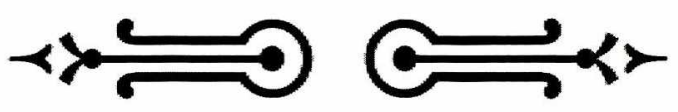